\title{
La filosofía y su historia. Un debate abierto*
}

\section{(Philosophy and its History. An Open Debate)}

\author{
María Cristina GonZÁLEZ y Nora STIGOL
}

Recibido: 14 de marzo de 2012

Aceptado: 20 de noviembre de 2012

\section{Resumen}

Las relaciones entre la filosofía y su historia han desencadenado, en las últimas décadas, una rica y amplia controversia. En este trabajo discutimos algunos aspectos de dicha controversia. En particular, cuestionamos la tesis frecuentemente sostenida de acuerdo con la cual el conocimiento de la historia de la filosofía es condición necesaria para la práctica filosófica y señalamos algunos supuestos -en su mayoría implícitos- que subyacen a esa afirmación.

Palabras claves: Filosofía, historia de la filosofía, diversos abordajes del pasado filosófico, supuestos implícitos.

\begin{abstract}
The relationships between philosophy and its history have been discussed at great length along the last decades. We analyze some aspects of this debate. Particularly, we question the thesis according to which to know history of philosophy is a necessary condition for doing philosophy. We disclose some assumptions, generally implicit ones, strongly related to it.

Keywords: Philosophy, history of philosophy, different perspectives of the philosophical past, implicit assumptions.

\footnotetext{
* Una versión preliminar de este trabajo fue leída en el Coloquio IIF-Sadaf 2011 organizado por el Instituto de Investigaciones Filosóficas (IIF) de la UNAM y por la Sociedad Argentina de Análisis Filosófico (SADAF) que tuvo lugar en Buenos Aires en octubre de 2011. Agradecemos muy especialmente las observaciones de Guillermo Hurtado Pérez (IIF-UNAM) y las de los participantes en la discusión que se suscitó a continuación. Agradecemos también los comentarios de uno de los informadores anónimos de la Revista de Filosofía.
} 
La filosofía contemporánea está atravesada por múltiples debates de distinta envergadura y amplitud. Uno de ellos concierne a la relación entre la filosofía y su historia. Esto se manifiesta en una extensa bibliografía en la que se exponen, defendiendo o atacando, posiciones muy diversas ${ }^{1}$. La importancia de esta controversia no es menor, Eduardo Rabossi (2008, p. 171) ha afirmado que "en lo que hace al futuro de la filosofía es, quizá, la polémica inmediata más trascendente".

La cuestión no es una cuestión meramente teórica, sino que tiene importantes consecuencias prácticas relativas, por ejemplo, a la enseñanza de la filosofía, a la organización de las instituciones, al diseño de los currícula, a las oportunidades laborales.

Las razones esgrimidas a favor de su relevancia abarcan diversas cuestiones que ameritan también atención, tales como: la naturaleza de la filosofía, la índole de la práctica filosófica, las características del conocimiento filosófico, la realidad del progreso filosófico, la noción misma de filósofo profesional, etc. No quedan a la zaga, además, cuestiones que tienen que ver con las relaciones entre la filosofía y otras prácticas y productos culturales (sean en el ámbito de las humanidades o en el de las ciencias), o las relaciones entre la historia de la filosofía y otras ramas de la historia, por ejemplo, la historia de las ideas.

Algunas veces se ha pensado, particularmente en la tradición analítica, que la filosofía, como sucede con otras disciplinas como las matemáticas, la biología o la química, debe ser desarrollada y enseñada sin atender a su historia. Otras veces, por el contrario y particularmente en la tradición continental, se ha sostenido que la filosofía es fundamental y esencialmente histórica, y, por lo tanto, no puede hacerse ni enseñarse sino históricamente. A nuestro juicio, y como sostuvimos en otra oportunidad (González y Stigol, 2012), las discusiones acerca de la relación entre la filosofía y su historia no deben ser pensadas como un capítulo dentro de la controversia más amplia que se ha dado entre la tradición analítica y la continental, en virtud, entre otras cosas, del disenso que existe respecto de los criterios para identificar una y otra 2 . Por lo demás, posiblemente, sea cierta la opinión de François Jaran (2011, p.172) cuando afirma que, aun cuando ambas tradiciones "se distinguen en cuanto a su relación con la historia", esos debates

ponen de manifiesto, entre otras cosas, la falsedad de los prejuicios que suele tener cada tradición sobre la otra: que la filosofía analítica ignora por completo el pasado de la filosofía o que la filosofía continental es incapaz de distinguir entre filosofía e historia de la filosofía.

\footnotetext{
1 Véase por ejemplo, la bibliografía que incluimos al final de este trabajo.

2 La controversia acerca de la existencia actual de dos tradiciones, la continental y la analítica, es motivo también de una extensa bibliografía y creemos que la que nos ocupa puede independizarse de ella.
} 
Posiblemente, también, sea acertada su afirmación $(2011$, p.172, 173) de que la cuestión de las relaciones entre la filosofía y su historia "constituye uno de los pocos temas donde pueda existir un debate fecundo entre las dos tradiciones"; en amplia consonancia con la afirmación de Rabossi citada anteriormente.

En el marco de estas controversias, se ha alegado con distintos argumentos y desde distintas perspectivas que dominar la historia de la filosofía es condición necesaria para la práctica filosófica (Ch. Taylor, 1984; L. Krüger, 1984; J. Cottingham, 2005; B. Williams 2002).

Decimos "desde distintas perspectivas", pues algunas veces se enfatiza el aspecto epistémico, es decir, se sostiene que no es posible comprender apropiadamente problemas y tesis filosóficas, si no se conoce su génesis y su desarrollo histórico. Así, por ejemplo Charles Taylor $(1984$, p. 31) sostiene: "Uno no puede ejercer la primera [la filosofía] sin ejercer también la segunda [la historia de la filosofía]. Dicho de otro modo, para comprender adecuadamente ciertos problemas, ciertas cuestiones, ciertas conclusiones, es esencial hacerlo genéticamente"3.

Otras veces, en vena hegeliana, y asumiendo una posición menos moderada, se enfatizan los aspectos ontológicos, y se afirma que la filosofía es por naturaleza esencial e intrínsecamente histórica. De ahí parece seguirse una afirmación aún más fuerte de acuerdo con la cual la filosofía es idéntica a su historia. Esta última tesis -la tesis de la identidad-, como toda tesis fuerte, resulta fácil de rebatir. Bastaría con mostrar, como sostiene Roy Mash (2001, p. 289) que no todo filósofo es de hecho un historiador ni estaría dispuesto a ser reconocido como historiador ni a la inversa, no todo historiador de la filosofía es ni estaría dispuesto a ser reconocido como filósofo. Por lo demás, parece considerablemente clara la distinción entre una obra o un libro de historia de la filosofía y una obra propiamente filosófica.

En nuestro medio ${ }^{4}$ se ha exagerado, en el mejor de los casos, la importancia de la historia de la filosofía para la filosofía o se ha reducido la última a la primera, identificándolas y, como consecuencia de ello, se ha confundido hacer y enseñar filosofía con hacer y enseñar su historia ${ }^{5}$. En consonancia con este punto de vista,

\footnotetext{
3 Tal vez esta afirmación sea válida para algunas áreas de la filosofía, por ejemplo en filosofía política y en ética. Véase al respecto B. Williams (2011). Por cierto, asumir la necesidad de estudios genéticos valga también para abordar cuestiones relativas a conceptos como los de modernidad, contemporaneidad, sujeto, Ilustración, subjetividad, cultura, secularización, etc.

${ }^{4}$ Nos referimos con ello al ámbito universitario en la Argentina y, creemos, que en general sucede lo mismo en la mayoría de los países latinoamericanos.

5 Una investigación sobre los planes y programas de estudios de las instituciones universitarias argentinas que hemos llevado a cabo en el marco del Proyecto UBACYT (Secretaría de Ciencia y Técnica de la Universidad de Buenos Aires) "Programa para el Mejoramiento de la Enseñanza de la Filosofía" fundamenta esta afirmación. Es de hacer notar que una investigación análoga podría hacerse respecto del número de contribuciones característicamente históricas respecto de las sistemáticas en reuniones académicas de carácter general (por ejemplo, congresos nacionales e internacionales de filosofía).
} 
no sólo se privilegian los cursos de historia de la filosofía sobre los cursos de materias sistemáticas, sino que, además estos últimos se presentan y se desarrollan también históricamente6.

Vale la pena advertir que esta tendencia a exagerar la importancia de la historia de la filosofía e identificarla con el quehacer propiamente filosófico se observa no solo en nuestro medio, sino que se extiende a otros ámbitos. Margaret Wilson, por ejemplo, ha señalado una importante expansión de libros y artículos con contenido histórico (en las décadas del 70 y del 80) que han dado lugar a una "próspera industria internacional" acerca de la cual Wilson examina sus posibles "frutos filosófi$\cos ^{\prime \prime}$ y Frank Ankersmit, editor del Journal of the Philosophy of History, al presentar los objetivos de la revista afirma ${ }^{8}$.

Ninguna explicación del conocimiento y la verdad puede ser considerada valiosa a menos que trate el tema de cómo nos relacionamos con nuestro pasado. El Journal of the Philosophy of History supone que la epistemología, la filosofía del lenguaje y la filosofía de la ciencia son incompletas si ignoran la historia.

Podría pensarse la identificación entre historia de la filosofía y filosofía o bien como una cuestión contingente, o bien como una cuestión normativa. En el primer caso, se trata de la constatación del hecho de que los filósofos conocen o han conocido la historia de la filosofía (cuestión empírica, que habría que probar en cada situación particular). En el segundo caso, la cuestión es si los filósofos deben conocer la historia de la filosofía.

Muchas veces, cuando se ofrecen razones de por qué los filósofos deben conocer la historia de la filosofía, se confunde precisamente lo que de hecho hacen o han hecho, con lo que se debe hacer. Si bien es cierto, como frecuentemente se sostiene, que los filósofos desarrollan sus ideas a partir de propuestas de colegas del pasado, esto es lo que de hecho sucede, pero de aquí no se sigue que deba ser así ni de que siempre sea así. Muchas veces los filósofos elaboran sus posiciones a partir de

\footnotetext{
6 Esta suerte de reduccionismo o identificación ha impregnado el modelo de enseñanza que en otros trabajos hemos denominado "modelo vigente" y que contrapusimos al modelo crítico que, a nuestro juicio resulta más adecuado, si se pretende formar filósofos profesionales capaces de elaborar posiciones propias y originales e insertarse en la comunidad filosófica internacional. Véase por ejemplo E. Rabossi, M. C. González, N. Stigol (1986); M. C. González y N. Stigol (1993); E. Rabossi, M. C. González y N. Stigol (1993).

7 Véase Margaret D. Wilson (1992, p.191): "En décadas recientes ha habido un inmenso flujo de libros y artículos sobre figuras y tópicos de la historia occidental de la filosofía. Esta notable y erudita producción ha acompañado, y ha sido reforzada por un amplio incremento de importantes proyectos editoriales y de traducción, y de promoción de foros para el intercambio oral que incluyen conferencias ad hoc de todo tipo y extensión, y de sociedades dedicadas al estudio de filosofías de individuos, períodos o tradiciones ideológicas". (La traducción es nuestra).

8 Enunciado de los objetivos de la revista Journal of the Philosophy of History.
} 
hipótesis científicas o de puntos de vista políticos o sociales o del sentido común y no de posiciones de filósofos del pasado.

Quizá no se trata de que los filósofos de hecho conozcan la historia de la filosofía ni que deban conocer historia de la filosofía, sino simplemente que es deseable que la conozcan.

El primer caso, como dijimos, es una cuestión empírica que habría que probar en cada situación particular y que requeriría alguna suerte de investigación que pusiera en evidencia que aquellos que conocen la historia de la filosofía producen filosofías mejores en algún sentido de "mejores". Hasta donde nosotras sabemos no se han hecho estudios en esta dirección.

Respecto del segundo caso, habría que responder a la pregunta de ¿por qué es necesario que los filósofos posean ese conocimiento? ¿Qué razones avalarían esa necesidad? Una respuesta afirmativa a esta pregunta parece darla Bernard Williams (2011 p. 226) cuando afirma:

En general, uno debe tomar extremadamente en serio la advertencia de Santayana de que quienes ignoran la historia de la filosofía están condenados a revivirla (no simplemente reinventar la rueda, sino a reinventar la rueda cuadrada).

Lo que nos proponemos en esta ocasión es discutir, precisamente, las respuestas a las preguntas que suscita la tesis de acuerdo con la cual quien pretenda filosofar debe conocer historia de la filosofía. Por otra parte, no es difícil proponer contraejemplos a la afirmación de que los filósofos deban conocer la historia de su disciplina para producir buena filosofía. Basta mencionar a los primeros filósofos griegos para quienes no había tal cosa como una historia de la filosofía a la cual recurrir o filósofos de la talla de Ludwig Wittgenstein que, al decir de Gilbert Ryle, estaba "orgulloso de no haber estudiado a otros filósofos" y pensaba que quien los estudiaba no eran auténticos filósofos 9 , o Jerry Fodor quien, se dice, se jacta de su ignorancia de la historia de la filosofía y de su habilidad para escribir "un libro sobre Hume sin conocer realmente nada acerca de él"10.

El tercer caso - que sea deseable conocer la historia de la filosofía- más próximo a nuestro punto de vista, requiere examinar los motivos que justificarían el conocimiento de la historia de la filosofía para la práctica filosófica y para su progreso -si es que lo hay - y, eventualmente, cuál sea su ventaja y conveniencia.

Nuestra hipótesis puede desglosarse en los siguientes puntos:

1. El conocimiento de la historia de la filosofía no es condición necesaria (ni mucho menos suficiente) para hacer filosofía. Aún reconociendo que haya historias

\footnotetext{
${ }^{9}$ Citado por Glock (2008a, p. 869-870).

10 Citado por Glock (2008a, p. 869).
} 
de la filosofia "mejores", a nuestro juicio, ni las mejores y más elaboradas constituyen condición necesaria para el ejercicio de la filosofía.

2. La historia de la filosofía y la filosofía son disciplinas independientes y autónomas, con diferentes metodologías, intereses y objetivos. Las diferencias metodológicas, de intereses y objetivos entre ellas merecen un tratamiento más atento que no abordaremos en el presente trabajo.

3. No asumimos una actitud à la Quine ni à la Harman ${ }^{11}$ respecto de la historia de la filosofía ni mucho menos compartimos el punto de vista de algunos a los que Jorge Gracia denomina "incompatibilistas"; ellos son quienes han visto en el estudio de la historia de la filosofía un efecto inhibitorio y pernicioso para la producción filosófica creativa. Descartes, de acuerdo con algunos autores (Gracia, 1992 y Daniel Garber, 2005), ejemplifica esta actitud hacia la historia de la filosofía. Recuérdese al respecto comentarios en el Discurso y en las Meditaciones acerca de las enseñanzas de sus antiguos maestros impartidas en las "más celebres y destacadas escuelas de Europa". Enseñanzas que Descartes rechaza a favor de alcanzar el conocimiento por sí mismo a través de su "propia razón y experiencia" y que no vacila en calificar como una "pérdida de tiempo".

4. Sostenemos una posición que, siguiendo a Hans-Johann Glock (2008) puede denominarse "historicismo pragmático" o "historicismo débil"12. Creemos que si bien el conocimiento de la historia de la filosofía no es imprescindible en la práctica filosófica, el filósofo puede beneficiarse con su estudio ${ }^{13}$. A nuestro juicio, el filósofo no se beneficia qua filósofo estudiando la historia de la filosofía como una

\footnotetext{
11 Recordemos la chanza de Quine cuando en una ocasión sostuvo que hay dos tipos de personas interesadas en la filosofía: Las que se interesan por la filosofía y las que se interesan por la historia de la filosofía, (Citado por A. MacIntyre (1984, p.59). B. Williams relata que en la puerta de la oficina de G. Harman podía leerse una nota en la que se decía "Diga no a la historia de la filosofía" (La traducción es nuestra)" (Citado por H-J. Glock 2008a, p. 869). El mismo episodio se cita también en B. Williams (2011, p. 226).

12 En un contexto distinto al nuestro - ya que su interés se centra casi exclusivamente en el ámbito de la filosofía analítica y en el debate acerca del supuesto desinterés de parte de los filósofos analíticos por la historia de la filosofía - Glock distingue tres maneras de concebir lo que denomina "historicismo" y por "historicismo" propone entender "cualquier posición que promueva el pensamiento histórico en filosofía y advierta contra la ignorancia o la distorsión del pasado" (Glock 2008b, p. 89). El historicismo, a juicio de Glock, puede ser pensado en un sentido fuerte como historicismo intrínseco o extremo de acuerdo con el cual no hay comprensión filosófica alguna si no en términos históricos o, dicho de otro modo, la filosofía es ipso facto histórica en sus métodos y en sus conclusiones (Glock 2008a, p.868.) (Glock 2008b, p. 90). El historicismo mainline o instrumental, más moderado que el anterior, afirma el carácter indispensable y necesario del estudio de la historia de la filosofía a la hora de elaborar tesis filosóficas sustantivas (Glock 2008a, p. 868) (Glock 2008b, p. 90). Por último, el historicismo pragmático constituye una forma más débil que reconoce a la historia de la filosofía como una herramienta útil y conveniente aunque no indispensable del quehacer filosófico.

13 Tal vez pueda ser de igual o de mayor utilidad el conocimiento de, por ejemplo, una disciplina artística o una ciencia, si ha de dedicarse a la estética o la epistemología.
} 
disciplina autónoma independiente de su situación presente, sino que se beneficia en la medida en que recurre al pasado partiendo de su problema aquí y ahora, cuando su actitud hacia su colega del pasado resulta similar a la de su actitud hacia su colega del presente buscando en él a alguien con quien dialogar (aunque sea a la distancia), con quien dirimir sus propios puntos de vista y elaborar una respuesta a sus problemas. Entendemos acá por "diálogo", el diálogo racional y crítico que, en palabras de Rabossi (2008, pp. 82-83), "es el ámbito propio y el motor de las discusiones y la producción filosóficas", en el que "la relación entre los participantes es simétrica" y cuya "meta no es el triunfo propio sino la búsqueda en común de la verdad, lo debido, lo justo o lo razonable". El filósofo busca en su colega (del pasado o del presente) a alguien que pueda señalarle sus propios errores argumentativos e indicarle nuevos caminos y nuevas estrategias argumentativas. No pretende análisis exegéticos ni comentarios acerca de los filósofos del pasado ni fidelidad a sus textos, esto parece ser una preocupación, en todo caso, para el historiador pero no para el filósofo. Por eso no nos inquieta una historia de la filosofía que resulte anacrónica. Más aún, como veremos más adelante, es esa la historia de la filosofía que preferimos defender a los efectos de su utilidad para la filosofía.

Creemos que, a la hora de analizar o de considerar la afirmación de que el conocimiento de la historia de la filosofía es condición necesaria para la actividad filosófica, es importante desbrozar el camino identificando los supuestos -en su mayoría implícitos- que subyacen a dicha afirmación y que, a nuestro juicio, es necesario poner en evidencia a fin de justificar la aceptación o no aceptación de ella. En nuestra opinión, quien defienda o cuestione dicha tesis está comprometido tanto con una concepción de la historia en general y de la historia de la filosofía en particular, como así también de la filosofía misma. En consecuencia, creemos que esos supuestos deben ser explicitados y examinados. ¿Cuáles son esos supuestos?

Ante todo, recordemos una ambigüedad frecuentemente 14 señalada de la expresión "historia" y por consiguiente también de la expresión "historia de la filosofía". La expresión "historia" refiere a un proceso en el que una serie de eventos pasados se han desarrollado efectivamente pero también refiere a la narración de tal proceso. Narración, que probablemente involucra no solo una dimensión descriptiva sino también interpretativa y evaluativa. Por cierto, la índole de la historia en general y - por qué no de la historia de la filosofía - ha sido y es objeto de intensos debates en el propio ámbito de la filosofía de la historia. "Historia" designa, además, una disciplina académica15.

\footnotetext{
14 Véase por ejemplo E. Rabossi (2008, p.149), F. Copleston (1992), J. Gracia (1992, p. 42 y siguientes). 15 J. Gracia (1992), por su parte, propone una elucidación del término "historia" que reconoce tres significados: 1) historia como colección de sucesos cronológicamente ordenados; 2) "historia" como relación (account) de una serie de acontecimientos pasados que contiene o debería contener referencias a sus causas y 3 ) el sentido de "historia" como disciplina de conocimiento (Véase p. 44 y siguientes).
} 
En un sentido trivial, la filosofía -como cualquier otra disciplina - es histórica en cualquiera de los sentidos señalados. En efecto, hay una sucesión de eventos desarrollados en el tiempo pasado que reconocemos como la historia de la filosofía. Y hay también una historia de la filosofía como la narración de esos acontecimientos y una historia de la filosofía como disciplina académica con problemas propios de toda disciplina académica, y que en el caso de la filosofía es un logro bastante tardío. Pero nada de esto, ni aun el hecho de que los conceptos y el lenguaje filosófico tengan un origen histórico, hace necesaria, a nuestro juicio, la relación de la filosofía con su historia.

Así como tropezamos con la ambigüedad de la noción de historia, enfrentamos también la ambigüedad de la expresión "filosofía". El término "filosofía" refiere tanto al proceso de producción filosófica (actos o acciones de ciertas personas - los filósofos-) como al resultado de ese proceso (los textos filosóficos). Es decir, "filosofía" designa un cierto tipo de actividad de naturaleza lingüística/conceptual que sigue ciertas reglas y que tiene por objetivo producir cierto tipo de saber, pero también alude al conjunto de escritos en los que se cristaliza y se objetiva ese saber. De este último podría decirse que es un saber que aspira a dar cuenta de las estructuras del mundo, de nosotros mismos y de nuestra ubicación e inserción en el mundo.

Así, por "filosofía" ha de entenderse tanto una actividad como el resultado de ella. Lo que hace el filósofo en tanto filósofo es llevar a cabo esa actividad y a través de ella producir filosofía en su segunda acepción como un corpus teórico que pretende dar cuenta de nuestra realidad y de la realidad en que vivimos. Según se ponga el acento en una u otra acepción posiblemente será distinta la respuesta respecto al carácter relevante o superfluo de la historia de la filosofía a la hora de comprender, encarar, resolver o disolver los problemas filosóficos ${ }^{16}$.

Pero no sólo cabe señalar esta ambigüedad; es bien sabido que no hay acuerdo entre los filósofos -aun entre los que se alinean en una misma tradición - acerca de la propia naturaleza de la filosofía. Antes bien es éste posiblemente el ámbito de un mayor desacuerdo. Hay quienes prefieren defender una versión naturalista de la filosofía -como un continuo con las ciencias naturales- otros, en cambio, defienden la idea de la filosofía como una disciplina humanista y otros como un conocimiento puramente conceptual y a priori etc. etc. Creemos que quien hace historia de la filosofía no sólo elige un modo o estilo de hacer historia, sino que también presupone -tácita o explícitamente- una concepción acerca de la filosofía que de modo más o menos manifiesto determina los criterios o parámetros que habrá de poner en juego para recoger y organizar los materiales disponibles. Sean estos eventos, autores, doctrinas, obras, argumentos o problemas a ser incluidos en su narración histórica.

Podrá argumentarse que de hecho, y dejando de lado algunas divergencias poco significativas, hay consenso acerca de cuáles son los eventos y los filósofos que

16 Véase por ejemplo J. Gracia (1992, p. 56) y J. Navarro Reyes (2010, Capítulo VIII). 
deberán incluirse (y excluirse) de una historia de la filosofía y cuáles sus obras, sus doctrinas, sus problemas. Recuérdese, además, que el historiador de la filosofía tropieza en su actividad con el hecho de que hay un canon de su disciplina concebido, o bien como una lista de autores o bien como un conjunto de problemas o tesis filosóficas que de alguna manera lo guiará en su tarea. Pero ¿cuándo, dónde y en virtud de qué valores o criterios se ha legitimado ese canon? Ciertamente, subyace a la historia de la filosofía un proceso evaluativo cuyos parámetros deberán ser explicitados. Nuevamente, estas cuestiones son objeto de reflexión de los filósofos de la historia.

Ahora bien, dado que lo que se historia es la filosofía y si es, como venimos diciendo, la concepción de esta disciplina la que orienta la tarea del historiador (aun cuando intervengan otros factores extrafilosóficos, sean éstos culturales, políticos, psicológicos o ideológicos), resulta que, para la tesis que estamos analizando, se desencadena una consecuencia poco deseable: una suerte de circularidad. Si esto es así, y si no se está dispuesto a aceptar explicaciones circulares, el carácter necesario atribuido a la historia de la filosofía para hacer filosofía deberá ser justificado de otro modo.

En tanto disciplina, la historia de la filosofía habrá de referirse a algún tipo de entidades; la pregunta a formularse es, entonces, ¿qué entidades serán concebidas como objetos a historiar? Dicho de otra manera, una historia de la filosofía ¿es una historia de los filósofos, de los sistemas, de las tesis, de los argumentos, de las ideas o conceptos filosóficos, de los problemas, etc. etc.? O ¿de todos ellos? Tal vez, ¿sólo de algunos? Y en ese caso ¿por qué unos o por qué otros? Pero, entonces, se abre una nueva cuestión. ¿Qué criterios permitirán distinguir las ideas/conceptos, tesis, problemas, argumentos filosóficos de las ideas/conceptos, tesis, problemas, argumentos en general o en particular de las ideas/conceptos, etc. científicos? Los conceptos de espacio y tiempo, por ejemplo, ¿son conceptos de la física o de la filosofía?; las tesis relativas a la adquisición del conocimiento ¿son tesis psicológicas o filosóficas?; Aristóteles o Newton ¿deberán ser incluidos en una historia de los filósofos o de los científicos? El argumento de la pobreza del estímulo ¿se inscribe en la historia de la lingüística o de la filosofía del lenguaje? ¿Cuáles son los parámetros -si los hay- que pueden ofrecerse a la hora de disponer en uno u otro lugar esos conceptos, argumentos, tesis, personas?

La concepción problemática de la historia de la filosofía originalmente formulada por Wilhelm Windelband, y especialmente defendida y difundida por John Passmore, ofrece una respuesta a una de las preguntas que acabamos de formular. De acuerdo con este punto de vista, son los problemas filosóficos los objetos que una historia de la filosofía debe historiar ${ }^{17}$.

17 Suele sostenerse (por ejemplo J. Navarro Reyes (2010, p. 117) que es en la tradición analítica donde prevalece la historia de la filosofía articulada en torno al planteamiento de problemas frente a una con- 
Creemos con Passmore que los filósofos no se proponen construir sistemas y doctrinas o acuñar nuevas ideas y conceptos, sino que procuran solucionar problemas (Passmore 1965, p.27). Secundariamente y con la finalidad de dar cuenta de esos problemas, elaboraran conceptos e ideas que estructuran en teorías y doctrinas. La filosofía es básicamente una actividad dirigida o enfocada a la resolución de problemas que el sentido común, la vida política, social, personal o la investigación científica plantean. De modo que son los problemas filosóficos los objetos que el historiador debería recoger.

La idea central que subyace a esta concepción es la suposición de que una teoría o doctrina no puede entenderse, si no se comprende cuál fue el problema que esa teoría intentaba resolver. Es la presión de un determinado problema lo que lleva al filósofo a elaborar sus propios puntos de vista. Esta forma de pensar la historia de la filosofía se contrapone a una historia pensada en términos de historia de filósofos, doctrinas y teorías encaminados hacia una supuesta "verdad filosófica"18.

Una versión de esta perspectiva sostiene que la historia de la filosofía, concebida como una historia de problemas, pone de manifiesto el carácter perenne de ellos; lo que ha sido esgrimido como un argumento a favor de la tesis que estamos examinando. En otro trabajo (González y Stigol, 2012), hemos considerado las dificultades que, en nuestra opinión, involucra la tesis de la perennidad de los problemas filosóficos. Dificultades relativas, por ejemplo, a una posible neutralidad en la formulación de los problemas; a la dependencia o independencia del problema respecto al contexto lingüístico, social, cultural, científico, histórico; a la cuestión del progreso en filosofía como así también relativas a la identificación e identidad del problema a lo largo del tiempo. Sostuvimos en aquella oportunidad que la noción misma de problema parece vaciarse de sentido, si, en efecto, como suele suceder, se piensa la perennidad en términos más fuertes que la mera recurrencia o el simple hecho de que sean similares o comparables, sino más bien en términos que involucran eternidad, irresolubilidad, ahistoricidad.

Dado que la filosofía es un producto cultural, es preciso acordar en qué momento habría de originarse su historia. ¿Cuándo se constituye como disciplina autónoma, si tal cosa puede decirse de la filosofía? ¿Dónde podríamos ubicar sus comienzos? La filosofía tal como hoy la practicamos surge tardíamente en la historia del pensamiento en tanto disciplina separada y distinguible de otros ámbitos del pensamiento, tales como el arte, la moral, la religión, la teología y principalmente de la

cepción más frecuente en la tradición continental en la que la historia se relata en torno a los filósofos y sus doctrinas.

18 Es posible que sea la oposición a o el desprestigio de los grandes sistemas metafísicos idealistas de finales del siglo XIX y principios del XX lo que haya ocasionado o redundado en el surgimiento y desarrollo de esta manera de pensar la historia de la filosofía, más cercana a las prácticas científicas (Véase L. Krüger, 1984, p. 103). 
ciencia. Precisamente con ésta, su separación es muy tardía, pues si bien en la cultura occidental para al menos algunas caracterizaciones (que por supuesto dependen de cómo concebir ciencia, filosofía e historia) ambas tienen orígenes remotos, su separación es relativamente reciente. Hasta avanzado el siglo XIX, "ciencia" y "filosofía" son expresiones sinónimas y por tanto no parece posible distinguir historia de la ciencia de historia de la filosofía. En siglos anteriores, bajo el título de "filosofía" se incluían trabajos no sólo de lógica y de metafísica sino también de geometría, aritmética, astronomía, medicina y teología. Recuérdese, además, la expresión tan usada en los siglos XVII y XVIII de "filosofía natural" que en definitiva no significaba sino lo que más tarde llamaremos ciencias naturales. En un artículo (1986, p.6) especialmente ilustrativo sobre este tópico, John Yolton sugiere utilizar las expresiones de "escritos interdisciplinarios" o "no disciplinarios" y mejor aún "escritos temáticos o problemáticos" para hacer referencia a los escritos que, a su juicio, confusamente se han incluido en otras épocas bajo la etiqueta de "filosofía".

Desde un punto de vista distinto al de Yolton, aunque perfectamente compatible con él, Rabossi (2008, p.13) defiende la tesis de que "La filosofía, lo que concebimos, practicamos y valoramos como filosofía, es una disciplina joven, sólo cuenta unos doscientos años". La larga historia que se le atribuye no es sino el resultado de la creación de una narración histórica que data también de 200 años atrás. No es éste el lugar para explayarnos sobre la posición o conjetura que Rabossi propone en su libro y con la que nosotras simpatizamos. Solo la mencionamos a los efectos de señalar una dificultad más a la hora de considerar la noción misma de historia de la filosofía.

Otra cuestión que reclama alguna aclaración es la siguiente: si de lo que se trata es de conocer la historia de la filosofía a los efectos de producir filosofía -tal como sostiene la tesis que estamos discutiendo-, cabe preguntarse en qué consiste ese conocimiento: ¿Se trata de leer los textos de un filósofo del pasado (remoto o reciente)? ¿Comentarlo? ¿Polemizar con él? ¿Estudiar el origen de un problema tal como fue formulado en el pasado? ¿Identificar relaciones de influencias entre los filósofos y/o las escuelas? ¿Citar filósofos del pasado en general o aquellos cuyas tesis nos interesan hoy para apoyar nuestros propios puntos de vista? ¿Encontrar padres antecesores ilustres que legitimen la importancia de nuestro problema actual o de nuestra tesis? ¿O se trata de dominar las narraciones producidas por historiadores de la filosofía? Y en este caso ¿cuál o cuáles narraciones se privilegiarán? Y ¿con cuanta profundidad y detalle? ¿O se trata de ubicar a nuestros colegas del pasado en sus contextos históricos? Posiblemente todo esto es valioso a la hora de elaborar una "buena" historia de la filosofía pero ¿qué de todo ello importa a la hora de hacer filosofía? 
Se requiere también elucidar la cuestión de la extensión o amplitud del pasado. Bajo la denominación de "historia", abarcamos tanto el pasado más remoto como el pasado más reciente 19 .

Habría que marcar una diferencia entre, por un lado, nuestra propia memoria y las de nuestros mayores presentes hoy y, por otro, la memoria entendida en términos de narraciones de quienes acceden a un pasado que no está basado en la memoria propia o en la de nuestros mayores, sino mucho más remota.

Caben pues algunas preguntas ¿cuál es la historia que se piensa necesaria para la práctica filosófica? ¿La reciente o la remota? Y además ¿cuál es el criterio -si lo hay - para distinguir entre una y otra? En definitiva, la línea divisoria entre el pasado remoto y el pasado reciente podría pensarse como una cuestión meramente arbitraria. En nuestra opinión, sería más interesante pensarla, siguiendo una sugerencia de Junqueira Smith y Bolzani Filho, no en términos meramente temporales sino más bien en términos de intereses y valores. Es decir, una teoría filosófica o un filósofo será tanto más remoto cuánto menos valioso resulte para la discusión y resolución de un problema que nos preocupa en el presente. Desde esta perspectiva, escriben los autores (2010 p. 356):

No es relevante saber cuándo un texto fue escrito y publicado, lo importante es saber si sus preguntas son también nuestras. Si lo son será un texto relevante para la reflexión filosófica, si no lo son, el texto será objeto de estudio histórico.

Examinemos a continuación una cuestión más general y compleja: ¿Qué entender por historia de la filosofía? Frente a esta pregunta, se desencadenan una multitud de respuestas bajo la forma de clasificaciones de modalidades o tipos de historia de la filosofía. En efecto, un análisis, aún superficial, permite advertir que es erróneo suponer que hay algo así como la historia de la filosofía y que es ella la que debe dominarse a la hora de hacer filosofía. De allí que el defensor de la tesis analizada deberá indicar cuál es la historia de la filosofía que, de acuerdo a su punto de vista, es necesaria y por qué esa y no otra.

La bibliografía sobre el estatus y la naturaleza de la historia de la filosofía pone en evidencia que hay una amplia variedad de géneros y modos de abordar el pasado filosófico, como así también distintos estilos de hacer historia de la filosofía.

19 Cabe hacerse eco de la afirmación de Margaret Wilson (1992, nota 6, p. 194) quien escribe: "Tomo como no problemático el que casi todos los filósofos que escriben hoy responden a la historia del discurso filosófico, en la medida que toman directamente en cuenta los trabajos publicados en los últimos años, e incluso en los primeros veinte años del siglo XX hasta finales del siglo XIX (dependiendo, en alguna medida, del tema). Es ciertamente posible, tener un enfoque principalmente histórico, digamos, Russell o Wittgenstein, pero el "carácter vital" de sus perspectivas no parece estar en discusión. La cuestión aguda, para nuestros propósitos presentes, es el grado en el cual muchos de los intereses y contribuciones de filósofos anteriores es pertinente para el esfuerzo filosófico contemporáneo" (la traducción es nuestra). 
Esta diversidad de modos de pensar y de hacer historia de la filosofía 20 depende, sin lugar a dudas, de la concepción de la filosofía que se sostenga, como así también de los objetivos propuestos y de la metodología apropiada para la obtención de dichos objetivos que deberían, en todo caso, ser explicitados. Veamos algunos de los puntos de vista que se han presentado.

i) El historiador podrá proponerse relatar con la mayor objetividad y lo más fielmente posible las tesis o posiciones de los filósofos del pasado, prescindiendo de toda crítica o polémica, y evitando en lo posible toda evaluación e interpretación ${ }^{21}$. Su metodología consistirá en parafrasear, glosar o resumir, con la mayor imparcialidad aquello que sus antecesores han sostenido. Sigue en pie, sin embargo, una cuestión no menor: ¿de qué criterios echará mano el historiador a la hora de elegir cuáles serán los filósofos y las tesis a exponer? ¿Valdrá acaso, acá, aquello de que la historia la escriben los ganadores? Es posible. Precisamente, una de las inquietudes que preocupan a los historiadores de la filosofía es la de "desenterrar" a aquellos que, por diversos motivos, pueden considerarse "los perdedores", entendiendo por ello aquellos filósofos que no forman parte del canon.

La historia que se elaborará será, así, fundamentalmente doxográfica, descriptiva y narrativa. Esta manera de hacer historia de la filosofía que, al decir de Richard Rorty, "provoca aburrimiento y desesperación" (1984, p. 83) no se propone comprender, a nuestro juicio, efectivamente la historia de la filosofía, sino sólo exponer lo que colegas del pasado han dicho. Por otra parte, no compartimos esta opinión de Rorty, pues no se trata de que una manera de hacer historia provoque "aburrimiento y desesperación", sino simplemente de que, en nuestra opinión, tal estilo de hacer historia de la filosofía no resulta interesante para hacer filosofía, en todo caso lo será para hacer historia.

ii) En contraste con el historiador doxográfico, los defensores de la llamada "reconstrucción histórica" (Rorty, 1984) ${ }^{22}$, si bien se proponen también descripciones objetivas e imparciales - no contaminadas de elementos presentes que conllevan puntos de vista anacrónicos-, pretenden, además, descubrir y entender la historia, hacer inteligibles, las preocupaciones, las creencias y los dichos de nuestros antecesores. Y esto solo es posible, de acuerdo con este punto de vista, haciéndolo en sus propios términos e incluyendo el estudio del contexto en el que elaboraron sus escritos. Incluir el contexto requiere todavía alguna clarificación. ¿Se trata del contexto histórico, social, político, económico etc. etc.? ¿Qué debería abarcar o cuán extendido debería ser el contexto a estudiar?

\footnotetext{
20 J. Gracia (1992) ofrece una taxonomía que comprende trece diferentes modos de aproximación a la historia de la filosofía. Véase también Véase también L. Braun, 1973; J. J. Gracia, 1998; G. Hatfield, 2005; J. Passmore, 1965 y 1967; R. Rorty, 1984.

21 No hay sin embargo historia que no sea, en mayor o en menor grado, interpretativa.

22 J. Gracia (1992, p. 241) llama a esta modalidad de hacer historia de la filosofía "The approach of the scholar".
} 
En muchos casos, este estilo puramente expositivo de relatar la historia de la filosofía hace de los textos del pasado una suerte de piezas de museo o documentos antiguos que merecen ser conservados y preservados y cuyo valor de verdad no está en discusión ${ }^{23}$. En la literatura sobre el tema, suele usarse la expresión "anticuarismo" para designar este punto de vista.

Algunas veces este modo de hacer historia de la filosofía va acompañado de una actitud de veneración y reverencia, o bien por un periodo de la historia pasada, o bien por algún filósofo que por diversos motivos -filosóficos o no- se hacen objeto de una suerte de devoción y nostalgia. Gracia alude a estos enfoques de la historia como el enfoque "nostálgico de la edad de oro" ("The golden age nostalgic") y el enfoque "romántico" ("the romantic"), respectivamente (Gracia 1992, p. 236 y p. 238). El historiador asume, entonces, los roles de un representante o vocero de una época o de un abogado defensor del punto de vista de su filósofo de preferencia.

Una dificultad en la que parece incurrir esta concepción de la historia de la filosofía y que fue señalada por María Isabel Santa Cruz (2003, p. 143), gira en torno a las siguientes preguntas: ¿es de hecho posible una reconstrucción histórica del pasado filosófico? ¿En qué medida es posible desprendernos de nuestro propio entorno, de nuestros propios puntos de vista y dialogar con nuestros antepasados en sus propios términos? ¿Es posible volcar las ideas del pasado en nuestros marcos conceptuales? A juicio de Santa Cruz todo intento en este sentido está condenado al fracaso: "Creer que podemos ser capaces de una reconstrucción histórica objetiva, escribe, suena a utopía por no decir ingenuidad" (p. 143). Si no podemos, como dice Santa Cruz, aproximarnos al pasado sino con nuestros propios esquemas conceptuales, entonces parece que el anacronismo es la única posibilidad de hacer historia. ¿Por qué no? Tal vez como dice Rorty la cuestión es solo ser consciente de ello. Por otra parte, creemos que el debate anacronismo versus no anacronismo es, en todo caso, un problema para el historiador de la filosofía, pero no para el filósofo en general, aunque sí puede serlo en tanto reflexione acerca de la naturaleza de la historia, es decir, en tanto filósofo de la historia.

iii) En contraste con esta actitud frente al pasado propia de un anticuario, otros historiadores asumen una actitud crítica y polémica que toma diversas formas ${ }^{24}$.

Su interés no es relatar objetivamente lo que otros dijeron, sino defender ideas o filósofos, criticar, evaluar, interpretar, debatir con sus antecesores considerándolos como sus pares. Podría decirse que su preocupación no es propiamente histórica sino fundamentalmente filosófica, su referencia al pasado no resulta de un inte-

\footnotetext{
23 Es ilustrativa la anécdota que relata H-J. Glock (2008a, p. 883). Luego de una clase sobre Aristóteles dictada por W. D. Ross, un estudiante le preguntó si Aristóteles estaba en lo correcto. A lo que Ross respondió: "No me formule tal pregunta. Yo solo trato de exponer lo que Aristóteles pensó. Averiguar si lo que él pensó es verdad o no, no es mi ocupación (business).

24 J. Gracia (1992 p., 253) incluye en esta modalidad de hacer historia de la filosofía seis variantes.
} 
rés por lo histórico sino por la filosofía misma. En sus formas, a nuestro juicio, más interesantes se trata de "reconstruir racionalmente", en términos de Rorty, los argumentos y puntos de vista de los filósofos muertos en nuestro propio términos. En rigor, no interesa lo que en efecto los filósofos del pasado han pensado y expresado; lo que importa es la verdad de las tesis sostenidas y, fundamentalmente, la pertinencia de las mismas para la discusión del problema que hoy, aquí y ahora, se plantea y se pretende resolver ${ }^{25}$. A este estilo de hacer historia de la filosofía - presentismo como opuesto al historicismo -, se le acusa de incurrir en anacronismos, de forzar a nuestros colegas del pasado a decir lo que no han dicho y a decirlo en un lenguaje que no les es propio. Y en algunos casos se le acusa de tergiversar lo que han sostenido. Es cierto que a veces las preocupaciones por problemas actuales pueden conducir o inclinar a los filósofos a escribir una historia distorsionada del pasado, pero eso parece ser un problema, en todo caso, para el historiador que busca fidelidad a los textos clásicos, aunque no para el filósofo que quiere elaborar una respuesta para resolver o examinar un problema que le preocupa, que se le plantea y que quiere zanjar. A nuestro juicio, es ésta, en todo caso, una mejor manera de valorar y estimar la tarea de nuestros ancestros. Parafrasearlos o repetirlos o resumir sus puntos de vista puede ser función del historiador, pero no contribuye de manera significativa, creemos, a hacer una "mejor" o más fructífera filosofía.

iv) Algunas veces el historiador de la filosofía asume una actitud que no resulta ser ni de la de un narrador fidedigno, ni la de un anticuario ni aún la de un colega polemizador o crítico, sino simplemente asume un rol de comentador. En este punto tomamos una distinción 26 trazada por Antonio Trajano (2004, p. 83) según la cual a veces se confunde bajo la denominación de "historia de la filosofía" dos tipos de investigaciones distintas. Una que consiste en "el comentario de obras filosóficas, entendido como estudios que no entran en los detalles de las conexiones de la obra comentada con la literatura filosófica anterior y/o subsiguiente" y otra, que denomina propiamente 'historia de la filosofía', "entendida en el sentido de estudios que cuentan el itinerario de las ideas y doctrinas en el tiempo y en el espacio, examinando, por consiguiente, el surgimiento y desarrollo de ésta o aquella doctrina o escuela en cierto lapso de tiempo, a veces más grande a veces más pequeño."

v) Otras veces la manera de entender la historia de la filosofía está vinculada con la noción de progreso filosófico que se pretenda defender (implícita o explíci-

\footnotetext{
25 "Leemos las filosofías del pasado en forma tal, resume A. MacIntyre esta posición, que ellas se tornen relevantes para nuestros problemas y nuestras empresas contemporáneas, transformándolas en la medida de lo posible, en lo que ellas habrían sido en caso de formar parte de la filosofía actual y minimizando o ignorando, o incluso presentando a veces erróneamente lo que se resiste a tal transformación porque se halla inextricablemente ligado con los elementos del pasado que lo tornan radicalmente distinto de la filosofía actual" (1984 p. 49).

26 Aunque el autor propone esta distinción para dar cuenta de la enseñanza de la filosofía en Brasil, consideramos que es útil tenerla en cuenta para mostrar distintas aristas profesionales.
} 
tamente). Así, por ejemplo, si se piensa el progreso como un camino en la dirección hacia una determinada meta generalmente vista como una verdad con mayúscula (piénsese en la filosofía de Hegel por ejemplo), o si se lo entiende como en la metáfora de Voltaire 27 como una suerte de movimientos de bailarines participando en un minuet, sin avanzar ni llegar a un determinado sitio, la historia será visualizada de diferentes maneras.

vi) Finalmente, la concepción de la historia de la filosofía de corte hegeliano no tiene como objetivo ni exponer, ni polemizar, ni comentar, ni aún mostrar el origen de los problemas filosóficos. Su finalidad es mucho más compleja. Es poner al descubierto la evolución que el Espíritu ha hecho desde sí mismo en el proceso de su autoconocimiento. Este objetivo, por cierto, resulta de una concepción muy peculiar de la filosofía -que no podemos abordar acá- según la cual su historia es vista bajo el signo del devenir teleológico de la Idea.

Por último, suele suponerse una asimetría entre historia de la filosofía y filosofía, por un lado, e historia de la ciencia y ciencia, por otro. Esta asimetría pone de manifiesto y revela, creemos, una manera peculiar de entender la relación entre la filosofía y su pasado, pues suele apoyarse en concepciones debatibles acerca de la naturaleza de los problemas científicos, de la práctica científica y de la noción de progreso atribuibles a tales prácticas. Esta cuestión la hemos tratado en otra oportunidad (González y Stigol, 2012).

Algunas reflexiones finales:

A lo largo de esta exposición hemos tratado de identificar los supuestos que creemos implícitos en la tesis que afirma que la historia de la filosofía es condición necesaria para el ejercicio de la filosofía. Es posible que aún haya otros presupuestos a desentrañar, si se quiere dar cuenta de este debate. O que en nuestra presentación hayamos errado en la interpretación de argumentos. Sin embargo, cabe adelantar algunas preguntas adicionales que nuestro análisis no contempló. Así, ¿por qué, según lo muestran algunas anécdotas mencionadas, se han asumido posiciones en ocasiones tan extremas? ¿Qué es lo que está en juego a la hora de considerar que esta discusión es importante? ¿Se trata de defender la filosofía ante nuevos desgajamientos? ¿Es por el contrario una defensa de una de las tantas ramas o subdisciplinas a que ha dado lugar la propia evolución de la historia como disciplina? ¿Se trata de un esfuerzo por legitimar lugares académicos, frente a la evolución de otras disciplinas sociales o humanísticas? ¿Se han agotado los argumentos para defender el lugar de la filosofía y del filósofo en el escenario cultural contemporáneo? ¿Es posible interpretar este debate como un enfrentamiento entre quienes aspiran y creen que es posible y legítima la independencia de la práctica filosófica y quienes prefieren el refugio protector en el pasado y la prescindencia de problemas actua-

27 Citado por A. Kenny (2005 p. 14). 
les? Intentar responder a estas preguntas vinculándolas con nuestras inquietudes iniciales es motivo de otro trabajo en el que será necesario incorporar datos de las prácticas filosóficas actuales. Pero el análisis de la tesis originaria sigue siendo un paso previo que creemos necesario dar.

\section{Referencias bibliográficas}

AgratTi, L. et al (1997): La enseñanza de la filosofía en la escuela secundaria. Aportes para un diagnóstico. Serie Extensión Universitaria, $\mathrm{n}^{\circ}$ 7. Buenos Aires, Facultad de Filosofía y Letras, Universidad de Buenos Aires.

Braun, L. (1973): Histoire de l'historie de la philosophie. Paris, Editions Ophrys. Benítez, L. (1988): "Reflexiones en torno a la metodología de la Historia de la Filosofía", en Dianoia 34, pp.181-193.

CoHen, L. (1986): “Doing Philosophy Is Doing Its History”, en Synthese, 67 (1), pp. 51-55.

Copleston, F. (1992): "Philosophy and its history", en Philosophy 67: 261, pp. $357-$ 365.

Cottingham, J. (2005): “Why do History of Philosophy?", en Sorrell, T \& G. A. J. Rogers (op.cit), pp. 25-42.

Curley, E. (1986): "Dialogues with the Dead", en Synthese, 67 (1), pp. 33-49.

GARBer, D. (2005): "What's Philosophical about the History of Philosophy", en Sorrell, T \& G. A. J. Rogers (op.cit.), pp.129-146.

Glock, H. J. (2008a): “Analytic Philosophy and History: A Mismatch?, en Mind, 117 (468), pp. 865-897.

GLock, H. J. (2008b): What is analytic philosophy?, Cambridge University Press. Glock, H. J. (1997): The rise of Analytic Philosophy, Oxford, OUP.

GonzÁlez, M. C., Stigol, N. (1993): "La Enseñanza de la Filosofía como la Enseñanza de una Técnica: el problema de los instrumentos de evaluación”, en Rabossi E. y Obiols G. (comps.) (1993), pp. 47-57.

GonzÁlez, M.C., RABossi, E. y Stigol, N. (1993): "Los seminarios en la enseñanza de la Filosofía", en Rabossi E. y Obiols G. (comps.), pp. 41-46

GonzÁlez, M. C. y Stigol, N. (2008): "La enseñanza de la filosofía y el canon filosófico. Algunas reflexiones”, en Pérez, Diana y L. Fernández Moreno (comps.), Cuestiones Filosóficas. Un homenaje a Eduardo Rabossi. Buenos Aires, Editorial Catálogos (2008), pp. 533-553.

GonzÁlez, M. C. y Stigol, N. (2012): “¿En qué se parecen la historia de la ciencia y la historia de la filosofía? Acerca de una supuesta falta de analogía”, en Ferreira, Juliana; Mesquita Hidalgo; Salvático, Luis; Silva, Cibelle (eds.) Filosofia e história da Ciência no Cone Sul. Encontro da AFHIC. (En prensa) 
Gracia, J. (1992): Philosophy and its History, State University of New York Press. Hay traducción al español (1998): La filosofia y su historia. México, IIFUNAM.

GraciA, J. (1987 a): "Problemas en la Enseñanza de la Filosofía en Ibero América", en Valdivia L. y Villanueva E. (comps.) op. cit., pp. 219-225.

Gracia, J. (1987 b): "La Filosofía y su Historia", en Revista Latinoamericana de filosofia, XIII (3), pp. 259-278.

Hatfield, G. (2005): "The History of Philosophy as Philosophy", en Sorrell, T. y A.J. Rogers (op.cit), pp. 83-128.

JARAN, F. (2011): "Cómo la tradición continental y la tradición analítica se enfrentan con la tradición filosófica", en Revista de Filosofía, Vol. 36 n 1, pp.17-192.

Junqueira Smith, P.y Bolzani Filho, R. (2010): "Filosofía e historia de la filosofía", en Nudler, O. (Ed.) op. cit., pp.349-372.

Kenny, A. (2005): “The Philosopher's History and The History of Philosophy", en Sorrell, T \& G. A. J. Rogers, op. cit., pp.13-24.

KRÜGER, L. (1984): “¿Por qué estudiamos la historia de la filosofía?”, en Rorty R., Schneewind, J.B. y Skinner, Q. (eds.), op. cit., pp.99- 126.

Leiter, B. (2004): The future for Philosophy, Oxford University Press.

MacInTYRe, A (1984): "La relación de la filosofía con su pasado", en Rorty R., Schneewind J.B. y Skinner Q. (ed.). op. cit., pp.49-68.

MASH, R. (2001): "How important for philosophers is the History of Philosophy?" en History and Theory, 26:3, pp 287-299.

Mulligan, K., Simons, P. and Smith, B. (2006): "What's Wrong with Contemporary Philosophy?". Preprint version of paper to appear in Topoi, 25 (1-2), pp. 63-67.

Navarro Reyes, J. (2010): Cómo hacer filosofía con palabras, Fondo de Cultura Económica. España.

NAthan, E., (1988): "Relevancia de la Historia de la Filosofía para la Filosofía", en Dianoia 34, pp. 201-205.

Nichols, R. (2006): "Why is the history of philosophy worth our study?", en Journal compilation, 2006 Metaphilosophy, 37 (1), pp.34-52.

Nudler, O. y Naishtat, F. (editores) (2003): El Filosofar hoy, Editorial Biblos, Buenos Aires.

Nudler, O. (Editor) (2010): Filosofía de la Filosofía. Enciclopedia Iberoamericana de Filosofía. Editorial Trotta, Madrid, España.

PASSMORE, J. (1965): "The Idea of a History of Philosophy", en History and Theory, 5 (5): pp. 1-32.

Passmore, J. (1967): "Philosophy, historiography of", en P. Edwards (ed.) Encyclopedia of Philosophy. New York/Londres, Macmillan, Vol.VI, pp. 226230. 
Rabossi, E., GonzÁlez, M.C. y Stigol N. (1986): "Un modelo de enseñanzaaprendizaje para un enfoque crítico de la Filosofía", en Revista de Filosofia y Teoría Política, 26-27, pp.158-163.

Rabossi, E. (1987): "Enseñar Filosofía y aprender a filosofar" en Valdivia L. y Villanueva E. (comps.), op. cit, pp. 201-208.

Rabossi, E. y Obiols, G. (1993): La Filosofía y el Filosofar: Problemas en su Enseñanza, Centro editor de América Latina.

Rabossi, E., (2000): "Psicólogos, Filósofos e interfases", en Análisis Filosófico, XX, (1-2), pp.199-211.

Rabossi, E. y Obiols, G. (2000): La enseñanza de la filosofía en debate. Ed. Novedades Educativas. Buenos Aires, México.

Rabossi, E. (2003): "El caso Rorty, un modelo para armar", en Nudler O. y Naishtat F. (ed.), op. cit., pp. 91-104.

Rabossi, E. (inédito), "Historiar la Filosofía y Enseñar a Filosofar".

Rabossi, E. (2008): Y en el comienzo Dios creó el canon. Biblia berolinensis. Buenos Aires, Gedisa.

RoRTY, R. (1984): "La historiografía de la filosofía: cuatro géneros" en Rorty, R. et al (1984) (op.cit), pp. 69-98.

Rorty, R., SchneEwind J.B. y SkinNer Q. (1984): Philosophy in History, Essays in the Historiography of Philosophy, Cambridge University Press. Cambridge. Versión en español: La Filosofía en la Historia. Ensayos de Historiografía de la Filosofía. (1990) Ed. Paidós Básica. Las citas en el presente trabajo pertenecen a esta edición.

RoRTY, R. (2002): Filosofia y futuro, Barcelona, Gedisa editorial.

SANTA CruZ, M. I. (2003): "Filosofía o Historia de la Filosofía: una falsa disyuntiva" en Nudler O. y Naishtat F. (eds.), op. cit., pp.139-150.

SchneEwind, J.B. (2006): Review, Sorrell, T \& G. A. J. Rogers (eds). Analytic Philosophy and History of Philosophy.OUP, 2005, en Notre Dame Philosophical Reviews.

Sorrell, T \& G. A. J. Rogers (eds). (2005): Analytic Philosophy and History of Philosophy. Oxford, OUP.

Striker, G (1999): "Why study the History of Philosophy?", en The Harvard Review of Philosophy, VII, pp. 15-18.

Stroll, A. (2002): La filosofía analítica del siglo XX, Siglo XXI Editores, Madrid, España.

TAYLOR, Ch. (1984): "La filosofía y su historia”, en Rorty, R. et al. (1984) op. cit., pp. 31-48.

Trajano Menezes Arruda, A. (2004): "La enseñanza de la filosofía: su situación en Brasil”, en Educación (1), pp. 83-87. 
Valdivia, L. y Villanueva, E. (comps.) (1987): La Filosofía y los problemas de su enseñanza, México, UNAM.

WiLliams, B. (2002): "Why Philosophy needs History?", London Review of Books. 17/10/2002: 7-9.

Williams, B. (2011): La filosofía como una disciplina humanística. México, FCE.

Wilson, C. (2005): "Is the History of Philosophy Good for Philosophy?", en Sorrell, T. y A. J. Rogers (op.cit.), pp. 61-82.

Wilson, M. (1992): "History of Philosophy in Philosophy Today, and the Case of the Sensible Qualities", en The Philosophical Review, 101, (1), pp. 191-243.

Yolton, J.W. (1986): "Is There a History of Philosophy? Some Difficulties and Suggestions", en Synthese, 67(1), pp. 3-21.

María Cristina González

Universidad Nacional de Rosario

Universidad Nacional de La Plata

mariacristinaguillermina@gmail.com

Nora Stigol

Universidad de Buenos Aires

Sociedad Argentina de Análisis Filosófico

nstigol@gmail.com 